\title{
ONCE-MONTHLY PALIPERIDONE PALMITATE AND ORAL ATYPICAL ANTIPSYCHOTICS IN THE TREATMENT OF PATIENTS WITH RECENT-ONSET SCHIZOPHRENIA
}

Ivona Šimunović Filipčić ${ }^{2}$, Katarina Matić ${ }^{2}$, Natko Gereš², Josefina Gerlach², Diana Prskalo-Čule², Tihana Zadravec Vrbanc², Vanja Lovretić2 ${ }^{2}$ Vinka Lesandrić3 ${ }^{\text {, Mirela Čelić }}{ }^{2}$, Vladimir Grošić2,4, Sandra Vuk-Pisk²,4, Žarko Bajić ${ }^{2}$, Igor Filipčić2,4,5

'Department of Psychiatry and Psychological Medicine, University Hospital Center Zagreb, Zagreb, Croatia; 2 Psychiatric Hospital "Sveti Ivan", Zagreb, Croatia; ${ }^{3}$ Department of psychiatry, General Hospital Vinkovci, Vinkovci, Croatia; ${ }^{4}$ Faculty of Medicine, Josip Juraj Strossmayer University of Osijek, Osijek, Croatia; ${ }^{5}$ School of Medicine, University of Zagreb, Zagreb, Croatia

Objective: To compare the efficacy of once-monthly paliperidone palmitate (PP1M) and atypical oral antipsychotics (AOA) in patients with recent-onset schizophrenia. Background: Atypical long-acting injectable (LAl) antipsychotics have become the mainstay of maintenance treatment for patients with schizophrenia. However, the majority of current clinical guidelines still do not provide clear recommendations for their use in patients with recent-onset schizophrenia.

Materials and methods: In this retrospective cohort study, we screened all patients admitted to the Centre for integrative psychiatry at the Psychiatric Hospital "Sveti Ivan", from January 2015 to December 2017. We defined the subpopulation of the patients with recent-onset schizophrenia by time since diagnosis ( $\leq 3$ years) and the number of previous psychiatric hospitalizations $(\leq 3)$. The psychiatric re-hospitalization within 12 months after the discharge was used as the measure of treatment efficacy.

Table 1. Participants' characteristics

\begin{tabular}{|c|c|c|}
\hline & $\begin{array}{l}\text { paliperidone } \\
\text { LAI }(n=91)\end{array}$ & $\begin{array}{l}2^{\text {nd }} \text { generation } \\
\text { oral }(n=144)\end{array}$ \\
\hline \multicolumn{3}{|l|}{ Gender } \\
\hline men & $68(74.7)$ & $75(52.1)$ \\
\hline women & $23(25.3)$ & $69(47.9)$ \\
\hline Age (years), median (IQR) & $27(22-33)$ & $30(22-38)$ \\
\hline \multicolumn{3}{|l|}{ Education } \\
\hline primary & $10(11.0)$ & $13(9.0)$ \\
\hline secondary & $62(68.1)$ & $105(72.9)$ \\
\hline university & $19(20.9)$ & $26(18.1)$ \\
\hline \multicolumn{3}{|l|}{ Work status } \\
\hline employed or student & $50(54.9)$ & $65(45.1)$ \\
\hline unemployed & $41(45.1)$ & $79(54.9)$ \\
\hline Comorbid chronic somatic illness & $13(14.3)$ & $20(13.9)$ \\
\hline $\begin{array}{l}\text { Age at psychosis onset (years), } \\
\text { median IQR }\end{array}$ & $27(22-33)$ & $29(22-38)$ \\
\hline Substance abuse & $44(48.4)$ & $45(31.3)$ \\
\hline \multicolumn{3}{|l|}{ Antipsychotic therapy } \\
\hline monotherapy & $21(23.1)$ & $77(53.5)$ \\
\hline 2 drugs combination & $51(56.0)$ & $53(36.8)$ \\
\hline$\geq 3$ drugs combination & $19(20.9)$ & $14(9.7)$ \\
\hline \multicolumn{3}{|l|}{ Other therapy } \\
\hline benzodiazepines & $52(57.1)$ & $85(50.0)$ \\
\hline mood stabilizers & $18(19.8)$ & $25(17.4)$ \\
\hline antidepressants & $15(16.5)$ & $32(22.2)$ \\
\hline anticholinergics & $40(44.0)$ & $24(16.7)$ \\
\hline \multirow{2}{*}{$\begin{array}{l}\text { hypnotics and sedatives } \\
\text { daily hospital within three months } \\
\text { from discharge }\end{array}$} & $6(6.6)$ & $21(14.6)$ \\
\hline & $14(15.4)$ & $22(15.3)$ \\
\hline
\end{tabular}

Results: A total of 235 recent-onset patients were identified, 91 treated with PP1M [median (IQR) age 27 (22-33)], and 144 treated with the AOA [median (IQR) age 30 (22-38)]. After the adjustment for a large number of possible sociodemographic and clinical confounders, patients treated with PP1M had $40 \%$ lower odds for the rehospitalization because of the psychosis relapse during the first 12 months after the discharge [OR=0.41 (CI95\% 0.18-0.92; $\mathrm{p}=0.035)$ ].

Table 2. Rehospitalization for relapse during the first 12 months

\begin{tabular}{|c|c|c|c|}
\hline & \multirow{2}{*}{ Relapse } & \multicolumn{2}{|l|}{ Adjusted } \\
\hline & & $\mathrm{OR}\left(\mathrm{CI}_{95 \%}\right)$ & $\mathrm{p}$ \\
\hline \multicolumn{4}{|l|}{ Study group } \\
\hline paliperidone LAI & $13(14.3)$ & $0.41(0.18-0.94)$ & 0.035 \\
\hline $2^{\text {nd }}$ generation oral & $33(22.9)$ & & \\
\hline \multicolumn{4}{|l|}{ Confounders controlled } \\
\hline \multicolumn{4}{|l|}{ Gender } \\
\hline men & 38 (20.9) & 1 & \\
\hline women & $16(16.0)$ & $0.53(0.24-1.17)$ & 0.114 \\
\hline Age (years) & & $1.28(0.91-1.80)$ & 0.161 \\
\hline \multicolumn{4}{|l|}{ Education } \\
\hline primary & $9(32.1)$ & 1 & \\
\hline secondary & 38 (18.9) & $0.39(0.13-1.14)$ & 0.084 \\
\hline university & $7(13.2)$ & $0.34(0.09-1.29)$ & 0.113 \\
\hline \multicolumn{4}{|l|}{ Work status } \\
\hline employed or student & $26(20.2)$ & 1 & \\
\hline unemployed & $28(18.3)$ & $0.67(0.33-1.35)$ & 0.260 \\
\hline \multicolumn{4}{|l|}{ Comorbid chronic somatic illness } \\
\hline no & $44(18.2)$ & 1 & \\
\hline yes & $10(25.0)$ & $0.79(0.27-2.30)$ & 0.664 \\
\hline Age at psychosis onset (years) & & $0.78(0.56-1.10)$ & 0.157 \\
\hline \multicolumn{4}{|l|}{ Substance abuse } \\
\hline no & $35(20.1)$ & 1 & \\
\hline yes & 19 (17.6) & $0.93(0.43-2.05)$ & 0.864 \\
\hline \multicolumn{4}{|l|}{ Antipsychotic therapy } \\
\hline monotherapy & $26(22.0)$ & 1 & \\
\hline 2 drugs combination & $19(15.4)$ & $0.81(0.36-1.82)$ & 0.614 \\
\hline$\geq 3$ drugs combination & $9(23.7)$ & $1.81(0.64-5.06)$ & 0.262 \\
\hline \multicolumn{4}{|l|}{ Other therapy } \\
\hline \multicolumn{4}{|l|}{ benzodiazepines } \\
\hline no & $18(14.8)$ & 1 & \\
\hline yes & $36(22.5)$ & $1.07(0.50-2.26)$ & 0.867 \\
\hline \multicolumn{4}{|l|}{ mood stabilizers } \\
\hline no & $39(17.5)$ & 1 & \\
\hline yes & $15(25.4)$ & $1.35(0.57-3.21)$ & 0.499 \\
\hline \multicolumn{4}{|l|}{ antidepressants } \\
\hline no & $43(18.8)$ & 1 & \\
\hline yes & $11(20.8)$ & $1.53(0.65-3.58)$ & 0.328 \\
\hline \multicolumn{4}{|l|}{ anticholinergics } \\
\hline no & 38 (18.6) & 1 & \\
\hline yes & $16(20.5)$ & $1.67(0.75-3.75)$ & 0.212 \\
\hline \multicolumn{4}{|l|}{ hypnotics and sedatives } \\
\hline no & $45(18.1)$ & 1 & \\
\hline yes & $9(27.3)$ & $1.67(0.47-3.38)$ & 0.653 \\
\hline \multicolumn{4}{|c|}{$\begin{array}{l}\text { daily hospital within three months } \\
\text { from discharge }\end{array}$} \\
\hline no & $50(20.7)$ & 1 & \\
\hline yes & $4(10.0)$ & $0.54(0.16-1.75)$ & 0.301 \\
\hline
\end{tabular}

CONCLUSIONS: The observed superiority of PP1M over AOA in the lower odds for rehospitalization during the 12 months after the hospital discharge, provides further evidence for the use of atypical LAI antipsychotics in the treatment of recent-onset schizophrenia. 